\title{
TELESCOPIC LINKAGES AND A TOPOLOGICAL APPROACH TO PHASE TRANSITIONS
}

\author{
MICHAEL FARBER ${ }^{\bowtie}$ and VIKTOR FROMM \\ (Received 5 July 2010; accepted 16 December 2010) \\ Communicated by S. Paycha \\ Dedicated to Alan Carey, on the occasion of his 60th birthday
}

\begin{abstract}
A topological approach to the theory of equilibrium phase transitions in statistical physics is based on the topological hypothesis, which claims that phase transitions are due to changes of the topology of suitable submanifolds in the configuration space. In this paper we examine in detail the antiferromagnetic mean-field $X Y$ model and study the topology of the subenergy manifolds. The latter can be interpreted mechanically as the configuration space of a linkage with one telescopic leg. We use methods of Morse theory to describe explicitly the Betti numbers of this configuration space. We apply these results to the antiferromagnetic mean-field $X Y$ model and compute the exponential growth rate of the total Betti number. Previous authors studied the Euler characteristic rather than the total Betti number. We show that in the presence of an external magnetic field the model undergoes a single 'total Betti number phase transition'.
\end{abstract}

2010 Mathematics subject classification: primary 55R80; secondary 82B26.

Keywords and phrases: Betti numbers, homology, linkage, configuration space, telescopic leg, phase transition, topological hypothesis.

\section{Thermodynamical phase transitions and topological changes in configuration spaces}

Equilibrium phase transitions are nonanalytic points of thermodynamic observables. Modern physics has accumulated experimental evidence that some new mathematical mechanisms might be relevant to phase transitions in certain systems. A recent mathematical approach to phase transitions is based on the topological hypothesis, which claims that at their deepest level phase transitions are due to changes of the topology of suitable submanifolds in the configuration space; see, for example, [4, 15] and references therein.

(C) 2011 Australian Mathematical Publishing Association Inc. 1446-7887/2011 \$16.00 
One knows that for a system with the Hamiltonian

$$
H=\frac{1}{2} \sum_{i=1}^{N} p_{i}^{2}+V\left(q_{1}, \ldots, q_{N}\right),
$$

assuming that $N$ is large, at any given value of the inverse temperature $\beta$, the effective support of the canonical measure is very close to a single equipotential hypersurface

$$
\Sigma_{v} \equiv\left\{q \in \Gamma_{N}: V(q)=v N\right\} .
$$

Here $\Gamma_{N}$ is the configuration space and $V: \Gamma_{N} \rightarrow \mathbf{R}$ is the potential. The topological hypothesis claims that changes of the topology of the hypersurfaces $\Sigma_{v}$ or of the manifolds with boundary

$$
\mathcal{M}_{v}=\left\{q \in \Gamma_{N}: V(q) \leq v N\right\}
$$

(as $N \rightarrow \infty$ ) are the reason for the singular behaviour of thermodynamic observables in phase transitions. One such observable is the configurational canonical free energy

$$
f_{N}(\beta)=-\frac{1}{N \beta} \log \int_{\Gamma_{N}} e^{-\beta V(q)} d q .
$$

In $[1,13]$ the quantity

$$
\sigma(v)=\lim _{N \rightarrow \infty} \frac{1}{N} \log \left|\chi\left(M_{v}\right)\right|
$$

involving the Euler characteristic $\chi\left(M_{v}\right)$, is studied as a function of $v$ and its nonsmoothness is related to the phase transitions. Recall that in the case where the potential $V$ is Morse, the Euler characteristic $\chi\left(M_{v}\right)$ is equal to the sum

$$
\sum_{i \geq 0}(-1)^{i} \mu_{i}(V)
$$

where $\mu_{i}(V)$ denotes the number of critical points of $V$ of Morse index $i$ lying in $M_{v}$. In the case where $V$ is Morse-Bott there is a similar formula,

$$
\sum_{Z}(-1)^{\operatorname{ind}(Z)} \chi(Z)
$$

where $Z$ runs over the critical submanifolds of $V$; see [6, Corollary 5.3].

In [10], a version of the topological hypothesis was proven for a class of short-range models (the Franzosi-Pettini theorem) with potentials of the form

$$
V(q)=\sum_{i=1}^{N} \phi\left(q_{i}\right)+\sum_{i, j=1}^{N} c_{i j} \psi\left(\left\|q_{i}-q_{j}\right\|\right),
$$

where $|\psi(x)|$ decreases faster than $x^{-d}$, with $d$ denoting the spatial dimension of the system. 
For the mean-field $k$-trigonometric model [1], which is characterized by the potential

$$
V_{k}(q)=\frac{1}{N^{k-1}} \sum_{i_{1}, \ldots, i_{k}=1}^{N}\left[1-\cos \left(q_{i_{1}}+\cdots+q_{i_{k}}\right)\right],
$$

where $q_{i} \in[0,2 \pi]$ are the angular variables, it was shown that phase transitions occur when $k \geq 2$, and the function $\sigma(v)$ in (1.2) is smooth if $k=1$ and nonsmooth if $k \geq 2$. Moreover, when $k \geq 2$, the function $\sigma(v)$ is nonsmooth precisely at the values of the energy at which the phase transitions occur. Thus, the purely topological quantity $\sigma(v)$ signals the absence or presence of a phase transition. This observation, as well as similar findings for other models, motivate the further study of the relations between thermodynamic phase transitions and the topology of the family $\mathcal{M}_{v}$. In the literature there are also results of a negative character concerning the topological hypothesis; see $[12,16]$.

\section{The antiferromagnetic mean-field $X Y$ model and the robot arm}

Christian Mazza pointed out a similarity between the study of the robot arm in [7, Sections 1.5 and 3.7] and the antiferromagnetic mean-field $X Y$ model [5, 14]. This observation was our point of departure; it motivated us to study a new class of linkages having one telescopic leg (that is, a leg with length variable in an interval) [8]; more details are given below.

The antiferromagnetic mean-field $X Y$-model has a potential of the form

$$
V=\frac{1}{2 N} \sum_{i, j} \cos \left(\theta_{i}-\theta_{j}\right)-h \sum_{i} \sin \theta_{i},
$$

where $\theta_{i} \in[0,2 \pi]$ are angular parameters (classical rotators) when $i=1, \ldots, N$. Here $h$ denotes an external magnetic field.

If $m$ denotes the complex magnetization vector

$$
\mathrm{m}=\frac{1}{N} \sum_{j=1}^{N} \exp i \theta_{j}
$$

and

$$
\mathfrak{m}_{0}=-i h \in \mathbf{C},
$$

then

$$
\left|\mathrm{m}+\mathrm{m}_{0}\right|^{2}=\frac{2}{N} V+h^{2} .
$$

We see that the sublevel set $\mathcal{M}_{v}$ given by (1.1) coincides with the set

$$
\mathcal{M}_{v}=\left\{q:\left|\mathrm{m}+\mathrm{m}_{0}\right|^{2} \leq 2 v+h^{2}\right\}
$$




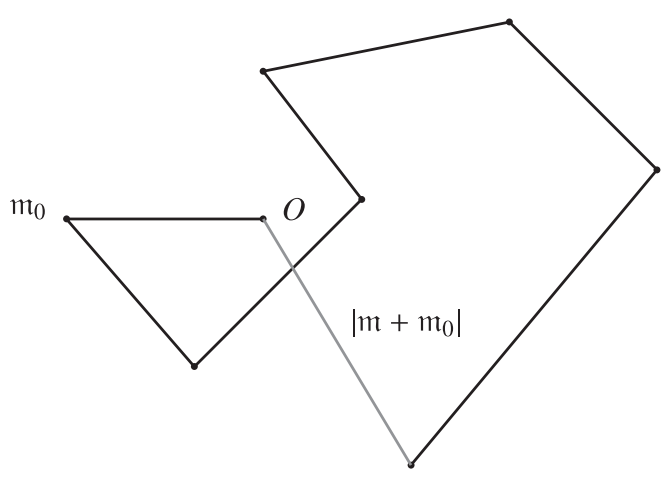

Figure 1. A robot arm.

In this case, $\Gamma_{N}$ is the $N$-dimensional torus $S^{1} \times \cdots \times S^{1}=T^{N}$; the symbol $q$ denotes a point $q=\left(e^{i \theta_{1}}, \ldots, e^{i \theta_{N}}\right) \in \Gamma_{N}$. When $h \in(0,1)$, the parameter $v$ may vary in the interval

$$
-\frac{1}{2} h^{2} \leq v \leq h+\frac{1}{2}
$$

However, when $h \geq 1$ (that is, there is a strong magnetic field), the interval of variation of $v$ is actually smaller,

$$
-h+\frac{1}{2} \leq v \leq h+\frac{1}{2}
$$

Differentiating (2.2) with respect to the angular variable $\theta_{j}$, we obtain

$$
\left\langle\frac{\partial \mathrm{m}}{\partial \theta_{j}}, \mathrm{~m}+\mathrm{m}_{0}\right\rangle=\frac{1}{N} \frac{\partial V}{\partial \theta_{j}},
$$

where the brackets $\langle\cdot, \cdot\rangle$ denote the Euclidean planar scalar product. Since clearly

$$
\frac{\partial \mathfrak{m}}{\partial \theta_{j}}=\frac{1}{N} e^{i\left(\theta_{j}+\pi / 2\right)},
$$

it follows that at a critical point of $V$ either $m+m_{0}=0$ or the vector $e^{i \theta_{j}}$ is parallel to $\mathrm{m}+\mathrm{m}_{0}$ for all $j=1, \ldots, N$. This implies that, when $h \neq 0$, at any critical point of $V$ lying outside the submanifold $m+m_{0}=0$ (the ground state), one has

$$
\theta_{j}= \pm \pi / 2 \text {, }
$$

that is, a critical configuration is collinear; it lies in the direction of the imaginary axis.

Consider now a robot arm with $N+1$ bars (as shown in Figure 1) where the length $\ell_{1}$ of first bar is $h$, and it points from the origin to the point $m_{0}$, while the remaining $N$ bars are of length $\ell_{j}=1 / N$, where $j=1, \ldots, N$, as described in [7, Section 1.5]. We assume that the initial point of the arm is fixed at the origin and the bars of the arm are connected to each other via revolving joints. If $\theta_{j}$ is the angle between the $j$ th bar and the horizontal $x$-axis, then $m+m_{0}$ in (2.1) is exactly the final position (the grip) of the arm. 
Clearly the length $\left|m+m_{0}\right|$ of the dotted line in Figure 1 is closely related to the value of the robot arm distance map studied in [7, Lemma 1.4]; the robot arm distance map is also described at the start of Section 4 below.

Lemma 1.4 from [7] deals with the shapes of the arm, that is, with the quotient of the configuration space with respect to the action of $\mathrm{SO}(2)$. The critical points of the robot arm distance map are the following: there is a critical submanifold $m+m_{0}=0$ (the ground state) that corresponds to the minimum of $V$. This submanifold has dimension $N-2$ and is diffeomorphic to the configuration space of a closed linkage with $N+1$ bars $(h, 1 / N, \ldots, 1 / N)$. The other critical points are of Morse type, and they correspond to collinear configurations of the arm, that is, $\theta_{i}= \pm \pi / 2$ when $i=2, \ldots, N+1$.

Translating this result into the language of thermodynamics, we obtain the biclustering phenomenon observed numerically in $[2,5]$. It consists of the statement that, under low temperature, some of the rotators are likely to point in a fixed direction $\psi_{0}$ with the remaining rotators pointing in the opposite direction $\psi_{0}+\pi$. This follows from the following three facts: first, that the canonical measure is concentrated near critical points of the energy $V$ as $T \rightarrow 0$; second, the critical points of the energy $V$ are the ground state $m+m_{0}=0$ and the Morse critical points corresponding to collinear configurations; and third, the ground state $m+m_{0}=0$ is highly degenerate.

Using (2.2), the manifold $\mathcal{M}_{v}=\{q: V(q) \leq N v\}$ can be interpreted mechanically as the configuration space of a linkage with $N+1$ legs of fixed lengths $h, 1 / N, \ldots$, $1 / N$ and one telescopic leg whose length varies between 0 and $\left(2 v+h^{2}\right)^{1 / 2}$. Recently, we studied linkages with telescopic legs whose length varies between two positive numbers [8]. In the next section, we give a modification of the arguments of [8] that allows for a computation of the Betti numbers in the important case where the telescopic leg is allowed to contract to zero.

\section{Telescopic linkages}

Motivated by the discussion of the previous section, we now study the topology of configuration spaces of telescopic linkages and calculate their Betti numbers.

Let $\ell=\left(\ell_{1}, \ell_{2}, \ldots, \ell_{n}\right)$ be a fixed vector with positive real entries, that is, $\ell_{i}>0$. Consider the variety of shapes $K_{\ell}$ of closed planar polygonal chains consisting of $n-1$ bars of fixed length (equal to $\ell_{1}, \ldots, \ell_{n-1}$ ) and a telescopic leg whose length may vary between 0 and $\ell_{n}$. Formally, $K_{\ell}$ is defined as follows. Consider the map $F: \mathbf{C}^{n} \rightarrow \mathbf{R}^{n}$ given by

$$
F\left(z_{1}, \ldots, z_{n}\right)=\left(\left|z_{2}-z_{1}\right|,\left|z_{3}-z_{2}\right|, \ldots,\left|z_{1}-z_{n}\right|\right) .
$$

Then $K_{\ell}$ can be defined as

$$
K_{\ell}=F^{-1}(A) / \mathrm{E}(2),
$$

where $A \subset \mathbf{R}^{n}$ is the closed interval connecting the points

$$
\ell^{-}=\left(\ell_{1}, \ldots, \ell_{n-1}, 0\right) \text { and } \ell=\left(\ell_{1}, \ldots, \ell_{n-1}, \ell_{n}\right) \text {, }
$$




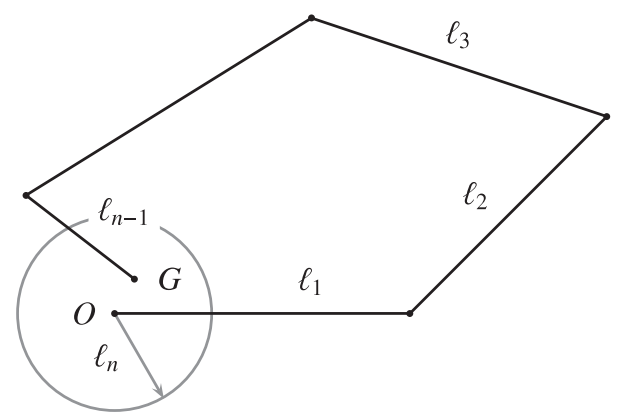

FIGURE 2. A configuration of the robot arm with the grip.

and $\mathrm{E}(2)$ denotes the group of orientation preserving isometries of the plane $\mathbf{C}$, acting diagonally on $\mathbf{C}^{n}$. Recall that

$$
M_{\ell}=F^{-1}(\ell) / \mathrm{E}(2) \subset K_{\ell}
$$

is the well-studied variety of shapes of closed polygonal chains with $n$ bars of lengths $\ell_{1}, \ldots, \ell_{n} ;$ see $[7,9]$.

The manifold $K_{\ell}$ can also be understood as the variety of all configurations of a robot arm with $n-1$ bars of length $\ell_{1}, \ldots, \ell_{n-1}$ such that the initial point $O$ is fixed, the first bar of length $\ell_{1}$ points in the direction of the $x$-axis, and the end point $G$ of the arm ('the grip') lies within a circle of radius $\ell_{n}$ with center at $O$ (see Figure 2).

Our goal in this paper is to compute the homology groups of $K_{\ell}$ explicitly as functions of the vector $\ell$ of metric data. In our previous paper [8], we considered configuration spaces of linkages having a telescopic leg not contractible to zero, that is, whose length may vary between two positive numbers. The results of the present paper complement those obtained in [8].

Recall that a vector $\ell=\left(\ell_{1}, \ldots, \ell_{n}\right)$ is said to be generic if $\sum_{i=1}^{n} \epsilon_{i} \ell_{i} \neq 0$ for all $\epsilon_{i}= \pm 1$.

PRoposition 3.1. If the length vector $\ell=\left(\ell_{1}, \ldots, \ell_{n}\right)$ is generic, then $K_{\ell}$ is a compact smooth $(n-2)$-dimensional manifold with boundary $\partial K_{\ell}=M_{\ell}$.

A proof is given in the following section.

It is clear that $K_{\ell}$ is diffeomorphic to $K_{\ell^{\prime}}$ if the vector $\ell^{\prime}=\left(\ell_{1}^{\prime}, \ldots, \ell_{n}^{\prime}\right)$ is obtained from from $\ell$ by permuting the coordinates $\ell_{i}^{\prime}=\ell_{\sigma(i)}$ provided that the permutation $\sigma:\{1, \ldots, n\} \rightarrow\{1, \ldots, n\}$ satisfies $\sigma(n)=n$. The $n$th coordinate, corresponding to the telescopic leg, plays a special role. It follows that, without loss of generality, one may always assume that $\ell_{1} \leq \ell_{2} \leq \cdots \leq \ell_{n-1}$.

Let $\ell=\left(\ell_{1}, \ldots, \ell_{n}\right) \in \mathbf{R}^{n}$ be a vector with positive coordinates. A subset $J \subset$ $\{1, \ldots, n\}$ is said to be short with respect to $\ell$ if and only if

$$
\sum_{i \in J} \ell_{i}<\sum_{i \notin J} \ell_{i}
$$


If the opposite inequality holds in (3.1) then $J$ is said to be long with respect to $\ell$. A subset $J \subset\{1, \ldots, n\}$ is called median if

$$
\sum_{i \in J} \ell_{i}=\sum_{i \notin J} \ell_{i} .
$$

Definition 3.2. Fix an index $i$ such that $\ell_{i}=\max \left\{\ell_{j}: j=1, \ldots, n-1\right\}$. We denote by $c_{k}(\ell)$ the number of $(k+1)$-element subsets $J \subset\{1, \ldots, n\}$ that contain $i$ but not $n$ and are short or median with respect to $\ell$; here $k=0,1, \ldots, n-2$. Further, for $k=$ $1, \ldots, n-2$, we denote by $d_{k}(\ell)$ the number of $(k+1)$-element subsets $J \subset\{1, \ldots, n\}$ that contain both $i$ and $n$ and that are short with respect to $\ell$.

It will be convenient to extend this definition by setting $d_{k}(\ell)=0$ for $k \leq 0$.

In [8] we introduced a symbol $\alpha_{k}(\ell)$ that is equal to the number of subsets of cardinality $k+1$ containing $n$ that are short with respect to $\ell$. One may express the numbers $c_{k}(\ell)$ and $d_{k}(\ell)$ introduced above through the quantities $\alpha_{k}$ as follows. Assume for simplicity that $\ell_{1} \leq \ell_{2} \leq \cdots \leq \ell_{n-1}$ (this can always be achieved by a permutation preserving the index of the telescopic leg). Then $d_{k}(\ell)$ is equal to $\alpha_{k-1}(L)$, where $L=\left(\ell_{1}, \ldots, \ell_{n-2}, \ell_{n-1}+\ell_{n}\right)$ is obtained by integrating the $(n-1)$ th and the $n$th legs. Further, if we assume that $\ell$ is generic, then $c_{k}(\ell)=\alpha_{k}\left(L^{\prime}\right)$ where $L^{\prime}=\left(\ell_{1}, \ldots, \ell_{n-2}, \ell_{n-1}-\ell_{n}\right)$.

Theorem 3.3. For a telescopic linkage described above with a generic vector $\ell$, the homology group $H_{k}\left(K_{\ell}\right)$ of the configuration space $K_{\ell}$ is free abelian, and its rank is equal to

$$
c_{k}(\ell)+d_{n-3-k}(\ell)
$$

when $k=0,1, \ldots, n-2$.

A proof is given in the following section.

Example 3.4. Consider the zero-dimensional Betti number $b_{0}\left(K_{\ell}\right)=c_{0}(\ell)+d_{n-3}(\ell)$. As above, we assume that $\ell_{1} \leq \cdots \leq \ell_{n-1}$. Clearly, $c_{0}(\ell)$ is equal to 1 if and only if the singleton $n-1$ is short or median with respect to $\ell$; otherwise $c_{0}(\ell)=0$. Moreover, when $n>3$, the number $d_{n-3}(\ell)$ is equal to 1 if and only if the set $\{n-2, n-3\}$ is long with respect to $\ell$; otherwise $d_{n-3}(\ell)=0$. We deduce that the manifold $K_{\ell}$ is disconnected if and only if

$$
\ell_{n-3}+\ell_{n-2}>\frac{1}{2} \sum_{i=1}^{n} \ell_{i} .
$$

COROllary 3.5. For $n>3$ the following conditions are equivalent.

(1) $K_{\ell}$ is disconnected.

(2) $K_{\ell}$ consists of two connected components.

(3) The set $\{n-3, n-2\}$ is long with respect to $\ell$.

(4) $K_{\ell}$ is diffeomorphic to the disjoint union $\left(T^{n-4} \times D^{2}\right) \sqcup\left(T^{n-4} \times D^{2}\right)$. 
Corollary 3.6. Suppose that $\ell_{n}>0$ is small in the following sense: for any choice of $\epsilon_{j}= \pm 1$ where $j=1, \ldots, n-1$, such that $\sum_{j=1}^{n-1} \epsilon_{j} \ell_{j}>0$, one has $\sum_{j=1}^{n-1} \epsilon_{j} \ell_{j}>\ell_{n}$. Then $K_{\ell}$ is homotopy equivalent to $M_{\ell^{\prime}}$ where $\ell^{\prime}=\left(\ell_{1}, \ldots, \ell_{n-1}\right)$.

We will skip the proofs of Corollaries 3.5 and 3.6 since they are analogous to the corresponding statements in $[8,11]$.

It is easy to see that under the assumptions of Corollary 3.6 the Betti numbers $b_{k}\left(K_{\ell}\right)$ coincide with those given by [9].

\section{Proofs of Proposition 3.1 and Theorem 3.3}

Consider the torus $T^{n-1}=S^{1} \times \cdots \times S^{1}$, and the quotient $W=T^{n-1} / \mathrm{SO}(2)$ with respect to the diagonal action of the rotation group $\mathrm{SO}(2)$. Clearly, $W$ can be identified with the torus of dimension $n-2$. Consider the function $f: W \rightarrow \mathbf{R}$ given by

$$
f\left(u_{1}, \ldots, u_{n-1}\right)=-\left|\sum_{i=1}^{n-1} \ell_{i} u_{i}\right|^{2} .
$$

Then $K_{\ell}$ can be identified with the preimage $K_{\ell} \simeq f^{-1}\left[a^{\prime}, 0\right]$, where $a^{\prime}=-\left(\ell_{n}\right)^{2}$.

We know that the critical points of $f$ consist of the preimage $f^{-1}(0)=M_{\ell^{\prime}}$, where $\ell^{\prime}=\left(\ell_{1}, \ldots, \ell_{n-1}\right)$, and finitely many Morse critical points corresponding to collinear configurations [9]. It follows that if the vector $\ell$ is generic then $a^{\prime}$ is a regular point of $f$ and therefore the preimage $f^{-1}\left[0, a^{\prime}\right]$ is a smooth manifold with boundary. This proves Proposition 3.1.

In the following arguments, we do not assume that $\ell$ is generic. In other words, $a^{\prime}$ is not necessarily a regular value of $f$. Let $a<a^{\prime}$ be a regular value of $f$ such that the interval $\left[a, a^{\prime}\right)$ contains no critical values.

Denote $W^{a}=f^{-1}(-\infty, a]$. Consider the long exact sequence

$$
\cdots \rightarrow H_{k+1}(W) \rightarrow H_{k+1}\left(W, W^{a}\right) \rightarrow H_{k}\left(W^{a}\right) \stackrel{j_{k}}{\longrightarrow} H_{k}(W) \rightarrow \cdots,
$$

where $j_{k}$ is induced by the inclusion $j: W^{a} \rightarrow W$. Using excision and Poincaré duality, we obtain

$$
H_{k+1}\left(W, W^{a}\right) \simeq H_{k+1}\left(f^{-1}[a, 0], \partial f^{-1}[a, 0]\right) \simeq H^{n-3-k}\left(f^{-1}[a, 0]\right) \simeq H^{n-k-3}\left(K_{\ell}\right) .
$$

Here we used the observation that $K_{\ell} \subset f^{-1}[a, 0]$ is a deformation retract.

Thus we obtain the short exact sequence

$$
0 \rightarrow \operatorname{coker}\left(j_{k+1}\right) \rightarrow H^{n-3-k}\left(K_{\ell}\right) \rightarrow \operatorname{ker}\left(j_{k}\right) \rightarrow 0 .
$$

Therefore to compute the cohomology of $K_{\ell}$ it is enough to find the kernel and cokernel of the homomorphism $j_{k}$; note that $H_{*}\left(W^{a}\right)$ and ker $j_{k}$ are torsion-free (see below) and therefore the exact sequence (4.1) splits.

Next we describe the homology of the manifold $W^{a}$ following [7-9]. For any subset $J \subset\{1, \ldots, n-1\}$, consider the subset $W_{J} \subset W \simeq T^{n-2}$ consisting of all configurations $\left(u_{1}, \ldots, u_{n-1}\right)$ such that $u_{i}=u_{j}$ for all $i, j \in J$. In other words, we 'freeze' all links 
labeled by indices in $J$ to be parallel to each other. It is clear that $W_{J}$ is diffeomorphic to a torus of dimension $n-1-|J|$.

The torus $W_{J}$ is contained in $W^{a}$, that is, $W_{J} \subset W^{a}$, if and only if $J$, viewed as a subset of $\{1, \ldots, n\}$, is long with respect to $\ell$. Indeed, let $p_{J}=\left(u_{1}, \ldots, u_{n-1}\right)$ be the configuration where $u_{i}=1$ for all $i \in J$ and $u_{i}=-1$ for all $i \notin J$. Then the maximum of the restriction $f \mid W_{J}$ is either 0 or $f\left(p_{J}\right)$; see [9, Lemma 8, Statement (4)]. The inequality $f\left(p_{J}\right) \leq a$ is equivalent to

$$
\sum_{i \in J} \ell_{i}>\frac{1}{2} \sum_{i=1}^{n} \ell_{i},
$$

which means that $J$ is long with respect to $\ell=\left(\ell_{1}, \ldots, \ell_{n}\right)$.

One may fix naturally orientations of $W$ and all submanifolds $W_{J}$ [8]. However, in this paper we will not need to deal with specific orientations and will assume that the manifolds $W_{J}$ and $W$ are somehow oriented. The class $\left[W_{J}\right] \in H_{k}(W)$ is then well defined where $|J|=n-1-k$.

If $k+k^{\prime}=n-2$ and $J$ and $J^{\prime}$ are subsets of $\{1, \ldots, n-1\}$ such that $|J|=n-1-k$ and $\left|J^{\prime}\right|=n-1-k^{\prime}$, then the intersection number $\left[W_{J}\right] \cdot\left[W_{J^{\prime}}\right]$ is either zero (if and only if $\left|J \cap J^{\prime}\right|>1$ ) or \pm 1 (if and only if $\left|J \cap J^{\prime}\right|=1$ ); see [9, Formula (33)]. Note that $k+k^{\prime}=n-2$ implies that $|J|+\left|J^{\prime}\right|=n$, that is, the subsets $J$ and $J^{\prime}$ must have a nontrivial intersection.

Without loss of generality, we may assume that $\ell_{1} \leq \ell_{2} \leq \cdots \leq \ell_{n-1}$.

One observes that the homology classes realized by the tori $W_{I} \subset W$, where $I$ runs over all subsets $I \subset\{1, \ldots, n-1\}$ of cardinality $n-1-k$ containing $n-1$, form a free basis of the homology group $H_{k}(W)$. We will write

$$
H_{k}(W)=A_{k} \oplus B_{k},
$$

where $A_{k}$ is generated by the homology classes $\left[W_{J}\right] \in H_{k}(W)$ where $n-1 \in J$ and $|J|=n-1-k$, such that $J$ is long with respect to $\ell$. The subgroup $B_{k}$ is generated by the classes $\left[W_{J}\right]$ with $J$ satisfying $n-1 \in J$ and $|J|=n-1-k$, such that $J$ is short or median with respect to $\ell$.

By $\left[9\right.$, Corollary 9], the homology classes $\left[W_{J}\right]$ of the submanifolds $W_{J}$, where $J$ runs over all subsets $J \subset\{1, \ldots, n-1\}$ of cardinality $n-1-k$ that are long with respect to $\ell$, form a basis of the free abelian group $H_{k}\left(W^{a}\right)$. This conclusion is based on the technique of Morse theory in the presence of an involution as developed in [9].

We will write

$$
H_{k}\left(W^{a}\right)=A_{k} \oplus C_{k},
$$

where $A_{k}$ is defined above and $C_{k}$ is generated by the classes $\left[W_{J}\right] \in H_{k}\left(W^{a}\right)$ such that $J \subset\{1, \ldots, n-2\}$ is long with respect to $\ell$ and $|J|=n-1-k$.

Note that $C_{k}=0$ if $k=0$.

Next we may analyse the homomorphism $j_{k}: H_{k}\left(W^{a}\right) \rightarrow H_{k}(W)$ induced by the inclusion $W^{a} \subset W$. It is obvious that $j_{k}\left[W_{J}\right]=\left[W_{J}\right]$ assuming that $\left[W_{J}\right] \in A_{k}$. 
We claim that if $\left[W_{J}\right] \in C_{k}$ then $j_{k}\left[W_{J}\right]$ is a linear combination of the classes $\left[W_{K}\right] \in A_{k}$. Indeed, we may write

$$
j_{k}\left[W_{J}\right]=\sum_{\left[W_{I}\right] \in A_{k}} a_{I} \cdot\left[W_{I}\right]+\sum_{\left[W_{K}\right] \in B_{k}} b_{K} \cdot\left[W_{K}\right],
$$

where $a_{I}, b_{K} \in \mathbf{Z}$. If $\left[W_{K}\right] \in B_{k}$, then the coefficient $b_{K}$ is equal to

$$
b_{K}= \pm\left[W_{J}\right] \cdot\left[W_{K^{\prime}}\right] \text {, }
$$

where $K^{\prime}$ is obtained from the complement of $K$ in $\{1, \ldots, n-1\}$ by adding $n-1$. If $b_{K} \neq 0$ then $J \cap K^{\prime}=\{j\}$ is a single element and hence $K$ is obtained from $J$ by removing $j \in J$ and adding $n-1$. However, $J$ is long with respect to $\ell$ and $\ell_{j} \leq \ell_{n-1}$, which imply that $K$ is long with respect to $\ell$ as well, and contradicts the assumption that $\left[W_{K}\right] \in B_{k}$.

From the exact sequence (4.1) we find that $H^{n-3-k}\left(K_{\ell}\right)$ is free abelian of rank

$$
\operatorname{rank} H^{n-3-k}\left(K_{\ell}\right)=\operatorname{rank} H_{n-3-k}\left(K_{\ell}\right)=\operatorname{rank} C_{k}+\operatorname{rank} B_{k+1} \text {. }
$$

Now we may calculate the ranks of $C_{k}$ and $B_{k+1}$. Examining the definition, we see that the rank of $C_{k}$ is equal to the number of $(k+1)$-element subsets $J \subset\{1, \ldots, n\}$ containing both $n-1$ and $n$ that are short with respect to $\ell$. In other words, rank $C_{k}=d_{k}(\ell)$. The rank of $B_{k+1}$ is equal to the number of $(n-2-k)$-element subsets $J \subset\{1, \ldots, n-1\}$ that contain $n-1$ and are short or median with respect to $\ell$, that is, rank $B_{k+1}=c_{n-3-k}(\ell)$. Substituting into (4.2), we obtain (3.2).

\section{The total Betti number phase transition in the antiferromagnetic mean-field $X Y$ model}

Consider again the antiferromagnetic mean-field $X Y$ model discussed in Section 2. According to the discussion at the end of Section 2 and by Theorem 3.3, the Betti numbers $b_{k}\left(\mathcal{M}_{v}\right)$ are equal to $c_{k}(\ell)+d_{n-3-k}(\ell)$, with the length vector $\ell$ being the following

$$
\ell=\left(\epsilon_{N}, \epsilon_{N}, \ldots, \epsilon_{N}, h,\left(2 v+h^{2}\right)^{1 / 2}\right),
$$

where $\epsilon_{N}=1 / N, n=N+2$ and $h$ denotes the magnetic field. We assume that $h$ is positive and constant, that is, independent of $N$.

Our goal is to examine the total Betti numbers

$$
b\left(\mathcal{M}_{v}\right)=\sum_{k=0}^{n-2} b_{k}\left(\mathcal{M}_{v}\right)=c(\ell)+d(\ell)
$$

and their rate of exponential growth

$$
\tau(v)=\lim _{n \rightarrow \infty} \frac{\log b\left(\mathcal{M}_{v}\right)}{n} .
$$

We want to investigate the possibility that singularities of this quantity, which might be more sensitive than (1.2), will allow detection of the phase transition. Here $c(\ell)$ 
denotes $\sum_{k} c_{k}(\ell)$; if $N$ is large, that is, if $N>1 / h$, then $c(\ell)$ is the number of all subsets of $\{1, \ldots, n\}$ that contain $n-1$ but not $n$ and that are short or median with respect to $\ell$. Similarly, $d(\ell)$ denotes $\sum_{k} d_{k}(\ell)$; when $N$ is large, $d(\ell)$ is the number of all subsets of $\{1, \ldots, n\}$ containing $n-1$ and $n$ that are short with respect to $\ell$.

Definition 5.1. We will say that the system undergoes a total Betti number phase transition at $v_{0}$ if the function $\tau$ given by the formula (5.2) is not analytic at $v_{0}$.

We motivate this definition by the topological hypothesis as presented in [12, Section V.A]. Namely, it is known that in many cases nonanalyticity of the function $\sigma$ mentioned in the introduction detects phase transitions of the system. The quantity $\tau$ is defined similarly to $\sigma$, but using the total Betti number rather than the Euler characteristic.

To illustrate the behaviour of $c(\ell)$ and $d(\ell)$, consider first the case where the magnetic field is strong, namely $h>1$. Then every subset containing both the indices $n-1$ and $n$ is long, and we obtain $d(\ell)=0$. On the other hand, the value of $c(\ell)$ in this case still depends on $v$.

Recall that the parameter $v$ varies in the interval $\left[a_{h}, b_{h}\right]$ where

$$
a_{h}= \begin{cases}-\frac{1}{2} h^{2} & \text { if } h \in(0,1], \\ -h+\frac{1}{2} & \text { if } h \in[1, \infty)\end{cases}
$$

and $b_{h}=h+1 / 2$; see Section 2. Let $p_{v}$ denote the quantity

$$
p_{v}=\frac{1}{2}\left(\left(2 v+h^{2}\right)^{1 / 2}-h+1\right) .
$$

It is easy to check that $0<p_{v}<1$ when $v \in\left(a_{h}, b_{h}\right)$. Besides, zero belongs to the interval $\left(a_{h}, b_{h}\right)$ and $p_{v}=1 / 2$ when $v=0$.

THEOREM 5.2. When $h>0$ and $v \in\left(a_{h}, b_{h}\right)$, the rate of exponential growth of the total Betti number (5.2) is equal to

$$
\tau(v)= \begin{cases}-p_{v} \log p_{v}-\left(1-p_{v}\right) \log \left(1-p_{v}\right) & \text { if } v \leq 0, \\ \log 2 & \text { if } v \geq 0 .\end{cases}
$$

In particular, the function $\tau$ and its first derivative are continuous but the second derivative of $\tau$ is discontinuous at 0 . In other words, the system undergoes a total Betti number phase transition at 0.

Proof. It will be convenient to use the notation

$$
S_{k}^{n}=\sum_{0 \leq i \leq k}\left(\begin{array}{l}
n \\
i
\end{array}\right) \text { and } R_{k}^{n}=\sum_{0 \leq i<k}\left(\begin{array}{l}
n \\
i
\end{array}\right) .
$$

Using formula (5.1), we may write

$$
b\left(\mathcal{M}_{v}\right)=S_{p_{v}(n-2)}^{n-2}+R_{\left(1-p_{v}-h\right)(n-2)}^{n-2} .
$$


If $v \leq 0$, then $1-p_{v}-h \leq p_{v} \leq 1 / 2$, and therefore

$$
\left(\begin{array}{c}
n-2 \\
{\left[p_{v}(n-2)\right]}
\end{array}\right)<b\left(\mathcal{M}_{v}\right)<2 \cdot S_{p_{v}(n-2)}^{n-2} \leq n \cdot\left(\begin{array}{c}
n-2 \\
{\left[p_{v}(n-2)\right]}
\end{array}\right) .
$$

We will use the following well-known asymptotic formula for the binomial coefficients,

$$
\left(\begin{array}{l}
n \\
m
\end{array}\right) \sim(2 \pi)^{-1}\left(\frac{n}{m}\right)^{m} \cdot\left(\frac{n}{n-m}\right)^{n-m} \cdot\left[\frac{m(n-m)}{n}\right]^{-1 / 2},
$$

which is valid when $n, m \rightarrow \infty$ and $n-m \rightarrow \infty$ (see [3, p. 4]). The meaning of the symbol $f(n) \sim g(n)$ is $\lim f(n) / g(n)=1$.

After some elementary calculations, the asymptotic formula above gives

$$
\lim _{n \rightarrow \infty} \frac{1}{n} \log \left(\begin{array}{c}
n-2 \\
{\left[p_{v}(n-2)\right]}
\end{array}\right)=-p_{v} \log p_{v}-\left(1-p_{v}\right) \log \left(1-p_{v}\right) .
$$

Now, the inequalities (5.4) imply the first part of (5.3).

If $v \geq 0$, then $p_{v} \geq 1 / 2$ and $S_{p_{v}(n-2)}^{n-2} \geq 1 / 2 \cdot 2^{n-2}=2^{n-3}$. In this case, therefore,

$$
2^{n-3} \leq b\left(\mathcal{M}_{v}\right) \leq 2^{n-1},
$$

which implies that $\tau(v)=\log$. This gives the second part of formula (5.3).

\section{Conclusions}

In this paper, we exploited an interpretation of the subenergy manifolds of the antiferromagnetic mean-field $X Y$ model as configuration spaces of linkages with one telescopic leg. Using Morse theory techniques, enriched with implications that stem from the presence of an involution, we gave a complete computation of the Betti numbers of the subenergy manifolds.

As an indicator of phase transitions, we studied the exponential growth rate of the total Betti number as opposed to the exponential growth rate of the Euler characteristic, as studied by the previous authors.

We showed by an explicit computation that, in the case of a nonvanishing magnetic field, there is a unique total Betti number phase transition in the antiferromagnetic mean-field $X Y$ model.

We hope that using the total Betti number instead of the Euler characteristic might provide a more sensitive tool for the study of different versions of the topological hypothesis. We suggest that the behaviour of the exponential growth rate of the total Betti number of the subenergy manifolds and its relationship to the physical properties of the system be examined in various models.

\section{Acknowledgement}

The authors are thankful to the anonymous referee for a number of valuable comments and suggestions. 


\section{References}

[1] L. Angelani, L. Casetti, M. Pettini, G. Ruocco and F. Zamponi, 'Topology and phase transitions: from an exactly solvable model to a relation between topology and thermodynamics', Phys. Rev. E 71 (2005), 036152 (112).

[2] M. Antoni and S. Ruffo, 'Clustering and relaxation in Hamiltonian long-range dynamics', Phys. Rev. E 52 (1995), 2361-2374.

[3] B. Bollobás, Random Graphs, 2nd edn, Cambridge Studies in Advanced Mathematics, 73 (Cambridge University Press, Cambridge, 2001).

[4] L. Casetti, M. Pettini and E. G. D. Cohen, 'Phase transitions and topology changes in configuration space', J. Stat. Phys. 111 (2003), 1091-1123.

[5] T. Dauxois, P. Holdworth and S. Ruffo, "Violation of ensemble equivalence in the antiferromagnetic mean-field $X Y$ model', Eur. Phys. J. B 16 (2000), 659-667.

[6] M. Farber, Topology of Closed One-Forms, Mathematical Surveys and Monographs, 108 (American Mathematical Society, Providence, RI, 2004).

[7] M. Farber, Invitation to Topological Robotics, Zurich Lectures in Advanced Mathematics (European Mathematical Society, Zurich, 2008).

[8] M. Farber and V. Fromm, 'Homology of planar telescopic linkages', Algebr. Geom. Topol. 10 (2010), 101-125.

[9] M. Farber and D. Schütz, 'Homology of planar polygon spaces', Geom. Dedicata 125 (2007), 75-92.

[10] R. Franzosi and M. Pettini, 'Theorem on the origin of the phase transitions', Phys. Rev. Lett. 92 (2004), 060601.

[11] M. Kapovich and J. L. Millson, 'On the moduli space of polygons in the Euclidean plane', J. Differential Geom. 42 (1995), 133-164.

[12] M. Kastner, 'Unattainability of a purely topological criterion for the existence of a phase transition in nonconfining potentials', Phys. Rev. Lett. 93 (2004), 150601.

[13] M. Kastner, 'Phase transitions and configuration space topology', Rev. Modern Phys. 80 (2008), 167-187.

[14] D. H. Lee, R. G. Caflisch and J. D. Joannopoulos, 'Antiferromagnetic classical XY model: a meanfield analysis', Phys. Rev. B 29 (1984), 2680-2684.

[15] M. Pettini, Geometry and Topology in Hamiltonian Dynamics and Statistical Mechanics, Interdisciplinary Applied Mathematics, 33 (Springer, New York, 2007).

[16] A. C. R. Teixeira and D. A. Stariolo, 'Topological hypothesis on phase transitions: the simplest case', Phys. Rev. E 70 (2004), 016113.

MICHAEL FARBER, Department of Mathematical Sciences, University of Durham, Durham DH1 3LE, UK e-mail: Michael.Farber@durham.ac.uk

VIKTOR FROMM, Department of Mathematical Sciences, University of Durham, Durham DH1 3LE, UK

e-mail: viktor.fromm@durham.ac.uk 